Discussion Paper No. 01-61

Die Evolution der bundesdeutschen Forschungs- und Technologiepolitik: Rückblick und Bestandsaufnahme

Andreas Fier und Dietmar Harhoff

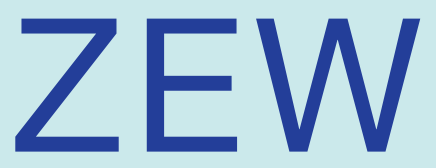

Zentrum für Europäische Wirtschaftsforschung GmbH

Centre for European Economic Research 
Discussion Paper No. 01-61

\title{
Die Evolution der bundesdeutschen Forschungs- und Technologiepolitik: Rückblick und Bestandsaufnahme
}

\author{
Andreas Fier und Dietmar Harhoff
}

Download this ZEW Discussion Paper from our ftp server:

ftp://ftp.zew.de/pub/zew-docs/dp/dp0161.pdf

Die Discussion Papers dienen einer möglichst schnellen Verbreitung von neueren Forschungsarbeiten des ZEW. Die Beiträge liegen in alleiniger Verantwortung der Autoren und stellen nicht notwendigerweise die Meinung des ZEW dar.

Discussion Papers are intended to make results of ZEW research promptly available to other economists in order to encourage discussion and suggestions for revisions. The authors are solely responsible for the contents which do not necessarily represent the opinion of the ZEW. 


\section{DAS WICHTIGSTE IN KÜRZE}

In Deutschland beanspruchen gleich zwei Ressorts, das Bundesministerium für Bildung und Forschung (BMBF) sowie das Bundesministerium für Wirtschaft und Technologie (BMWi) die Zuständigkeit für die deutsche Innovationspolitik. Dieses für den Bund offensichtlich bedeutsame Politikfeld eröffnet zum einen die Frage, auf welchen Prinzipien die gegenwärtige Forschungs- und Technologiepolitik aufgebaut ist und zum anderen, wie es gelingen soll, die wissenschaftlichen und wirtschaftlichen Rahmenbedingungen für FuE-Aktivitäten zu verbessern.

Dieser Beitrag widmet sich der industrieökonomischen Innovationsforschung und der Umsetzung ihrer Ergebnisse in der Wirtschaftspolitik. Ausgangspunkt ist ein Rückblick auf theoretische Konzepte, die seit den grundlegenden Studien von Arrows (1962) in der Literatur entwickelt worden sind. Dazu gehören die Entwicklung von Patentrennenmodellen, Modellen präemptiven Verhaltens und Überlegungen zur Funktionsweise von Wissensexternalitäten. Gerade das Konzept der "absorptiven Kapazität", Ende der 80er Jahre von Cohen und Levinthal vorgeschlagen, hat sich nicht nur in der volkswirtschaftlichen Forschung als wichtig erwiesen, sondern auch in der Betriebswirtschaftslehre. Den Fortschritten in der Wissenschaft steht eine Entwicklung in der Forschungs- und Innovationspolitik gegenüber, die nur mäßig von ökonomischen Konzepten beeinflusst worden ist. Nach Darstellung des Entwicklungsverlaufs der bundesdeutschen Innovationspolitik kommt eine Analyse des Förderinstrumentariums zu dem Schluss, dass insbesondere die Wirkungsforschung in Deutschland noch unterentwickelt ist und vor neuen Herausforderungen steht. 


\title{
Die Evolution der bundesdeutschen Forschungs- und Technologiepolitik: Rückblick und Bestandsaufnahme
}

\author{
von \\ Andreas Fier \\ Zentrum für Europäische Wirtschaftsforschung (ZEW) \\ Dietmar Harhoff \\ Ludwig-Maximilians-Universität München, ZEW und CEPR
}

\begin{abstract}
We consider the development of German federal research and technology (R\&T) policies since the 1960s and sketch the evolution of today's highly differentiated and complex set of policy instruments. Advances from economic theory and empirical results are reflected in this evolution, but have not necessarily been the driving force. In some instances, innovative policy instruments have been introduced in order to accomodate the state of the art in economic analysis; in other cases, such innovations have preceded a thorough analysis of the respective policy instruments. A major point of concern is the lack of comprehensive evaluation and cost-benefit analyses in R\&T policies. In this regard, German policy practice lags behind well-established procedures in other countries.
\end{abstract}

Keywords: Innovation, Public R\&D Subsidies, Policy Evaluation

JEL Class: O31, H32, C24

Adresse: Zentrum für Europäische Wirtschaftsforschung GmbH

Postfach 103434

D - 68034 Mannheim

Tel: $\quad+49 /(0) 621 / 1235-295$

Fax: $\quad+49 /(0) 621 / 1235-170$

E-mail:_fier@zew.de, harhoff@bwl.uni-muenchen.de

Für hilfreiche Hinweise und Kommentare danken wir Jürgen Egeln und Christian Rammer. 


\section{$1 \quad$ Einleitung}

In der Forschungs- und Technologiepolitik (FuT-Politik) hat der Innovationsbegriff Hochkonjunktur. Im Zuge des Neuzuschnitts der Ressorts Bildung und Forschung (BMBF) sowie Wirtschaft und Technologie (BMWi) beanspruchen seit 1998 gleich zwei Bundesministerien die Zuständigkeit für die deutsche Innovationspolitik. Während das BMBF traditionell seine Arbeitsschwerpunkte in der Förderung der Grundlagenforschung, der Vorsorge- und Bildungsforschung, in Schlüsseltechnologien sowie in der Verkehrs- und Raumfahrtforschung sieht, konzentriert sich das BMWi nunmehr auf die Innovationsfähigkeit des Mittelstandes, auf die Energieforschung und die zivile Luftfahrt. Die Adressaten beider Ministerien sind Wissenschaft und Wirtschaft, zunehmend aber auch Verbände, Industrie- und Handelskammern, internationale Organisationen und andere Akteure des Innovationssystems. Das Ziel der FuT-Politik ist es, sowohl die wissenschaftlichen und wirtschaftlichen Rahmenbedingungen für FuE-Aktivitäten zu verbessern als auch die internationale Wettbewerbsfähigkeit deutscher Unternehmen zu stärken. Im Mittelpunkt dieser Politik steht die Beschleunigung des Wissens- und Technologietransfers sowie die schnellere Umsetzung von FuE-Ergebnissen in marktfähige Produkte. ${ }^{1}$ Zur Durchsetzung dieser Ziele bedient sich der Staat verschiedener Förderinstrumente, die in diesem Beitrag diskutiert werden. Zuwendungen, Darlehen, Bürgschaften, die Absicherung von Kreditrisiken oder eine verbesserte Infrastruktur sollen FuE-Aktivitäten ermöglichen und Innovationen förderlich sein. Dabei werden seit den neunziger Jahre auch neue Förderkonzepte erprobt. „Inno“-Programme mit den bezeichnenden Namen „InnoNet“, „InnoRegio“ oder „ProInno“ sollen Anreize geben, um alle Akteure des Innovationssystems zu Kooperationen und einer verstärkten Innovationstätigkeit anzuregen.2 Der Staat verstehe sich dabei nur als Moderator, der durch das Aussetzen von Preisgeldern und Vorgabe einer breit defi-

1 So ist in BMWi/BMBF (2000, 3) nachzulesen: „Die Bundesregierung räumt der FuT-Politik einen herausragenden Stellenwert ein. Ziel ist es, die Voraussetzungen für Innovationen und technischen Fortschritt nachhaltig zu verbessern und damit vor allem die Innovationsfähigkeit der mittelständischen Wirtschaft zu unterstützen. Zugleich sollen neue Forschungsergebnisse schneller ihren Weg in den Markt finden. Deshalb werden für Forschung und Innovationen mehr Fördermittel bereitgestellt.“

2 Hierzu BMWi (2001, 27): „Mit dem Programm InnoNet sollen KMU, - einschließlich des Handwerks - und Forschungseinrichtungen für eine stärkere Zusammenarbeit gewonnen werden, um Ergebnisse der Forschung und Entwicklung schneller in marktfähige Verfahren und Dienstleistungen umzusetzen mit dem Ziel, die Wettbewerbsfähigkeit der Unternehmen zu stärken.“ 
nierten Förderthematik Anreize für private Akteure schafft, stärker auf Innovation zu setzen.

Diese Programmatik wirft viele Fragen auf, insbesondere im Hinblick auf die ,neuen Systemansätze“ der FuT-Politik. Kennzeichnen diese Ansätze tatsächlich den Übergang von der klassischen FuE-Förderung zu einer Innovationsförderung, die mit relativ schwachen staatlichen Eingriffen verbunden ist? Auf welchen theoretischen Prinzipien ist die gegenwärtige Forschungs- und Technologiepolitik, gerade nach ihrer Neuausrichtung, aufgebaut? Welche Berechtigung hat sie aus wirtschaftswissenschaftlicher Sicht?

Im Zusammenhang mit der aktuellen Vergabepraxis öffentlicher FuEFördermittel befassen wir uns mit vier Fragenkomplexen:

- Wie haben sich die theoretischen Konzepte entwickelt, der sich die FuT-Politik im Laufe der Jahre bedient hat?

- Auf welche empirische Evidenz kann zurückgegriffen werden, um die FuTPolitik wissenschaftlich zu beurteilen?

- Worin unterscheiden sich die klassischen forschungspolitischen Ansätze von neueren (sogenannten) innovationspolitischen Maßnahmen?

- Welcher Handlungsbedarf leitet sich aus der veränderten FuT-Politik für die Wissenschaft bzw. Wirkungsforschung ab, insbesondere bezüglich der Ergänzung der theoretischen Grundlagen und der empirischen Evaluation von Politikmaßnahmen?

Im zweiten Abschnitt werden die wichtigsten Erkenntnisse der theoretischen und empirischen Innovationsforschung in ihrem Entwicklungsverlauf der letzten 40 Jahre skizziert. ${ }^{3}$ Wir beginnen unsere Betrachtung mit den Studien, die 1960 bei einer Konferenz des National Bureau of Economic Research vorgestellt worden waren (Nelson 1962). Wir gehen der Frage nach, in welcher Form die damals angesprochenen Forschungsthemen sich weiter entwickelt haben, und in welcher Form sie durch neue Ansätze, aber auch neue Forschungsfragen ergänzt wurden. Daran

3 Wir beschränken uns dabei auf die auf volkswirtschaftliche Implikationen ausgerichteten Forschungsergebnisse, wenngleich betriebswirtschaftliche und volkswirtschaftliche Innovationsforschung längst nicht mehr strikt trennbar sind. Eine sinnvolle Charakterisierung von Innovationsprozessen in Unternehmen ist eine wichtige Voraussetzung für theoretische und empirische Arbeiten in diesem Feld, unabhängig davon, ob sie auf betriebs- oder volkswirtschaftliche Handlungsempfehlungen abzielen. 
schließt sich ein Überblick über besonders wichtige empirische Ergebnisse an, die diese Theorien prüfen und dazu beitragen, das Wissen über Innovationsprozesse in der Volkswirtschaft zu erweitern.

Der dritte Abschnitt enthält eine detaillierte Darstellung der Entwicklung der FuT-Politik seit den 60er Jahren und beschreibt die Ausgestaltung klassischer und neuer Förderansätze der FuT-Politik. Unsere Betrachtung zeigt, dass die FuTPolitik sehr wohl auf den wirtschaftspolitischen Diskurs reagiert hat, in einigen Fällen aber einer Eigenlogik gefolgt ist, die die Innovationsforschung vor Herausforderungen stellt. Hier sind sowohl theoretische als auch empirische Beiträge gefragt, um FuT-Politikmaßnahmen zu untermauern bzw. zu bewerten.

Wir nehmen unsere Beobachtungen in Abschnitt 4 zum Anlass, abschließend einige der wissenschaftlichen Herausforderungen zu benennen, die entweder immer noch bestehen oder mit den neueren innovationspolitischen Maßnahmen erstmals einhergehen. Damit verbunden ist die Aufforderung an die FuT-Politik, sich stärker als bisher auf den Diskurs mit der Innovationsforschung einzulassen - insbesondere im Bereich der Evaluation von FuT-Politikmaßnahmen. Gerade die „neuen Systemansätze“, die verstärkt auf eine Beschleunigung der Vermarktung von Innovationsergebnissen abzielen, bedürfen dringend einer empirischen Evaluation.

\section{Meilensteine der theoretischen und empirischen Innovationsforschung}

Um die weiter unten beschriebene Evolution der Forschungs- und Technologiepolitik in einen wirtschaftswissenschaftlichen Bezugsrahmen zu setzen, bedarf es zunächst einer Übersicht über Meilensteine der theoretischen und empirischen Forschung. Dabei wollen wir der langen Reihe von Artikeln, die die theoretische Rechtfertigung für staatliche Eingriffe diskutieren, keinen weiteren hinzusetzen, sondern wichtige Resultate kurz in chronologischer Reihenfolge nennen. ${ }^{4}$

Als Ausgangspunkt dient uns dabei der Konferenzband ,The Rate and Direction of Inventive Activity“, der 1962 von Richard Nelson herausgegeben wurde und die Papiere einer Konferenz zusammenfasste, die im Frühjahr 1960 an der University of Minnesota stattgefunden hatte. Diese Sammlung von Studien ist bemerkenswert, da mit dem Konferenzband eine Forschungsagenda vorgelegt wurde, die auch heute noch - zumindest im Hinblick auf die angesprochenen Forschungsfragen - aktuell ist. Der bekannteste Beitrag ist die Untersuchung von Arrow zur Allokation von FuE-Ressourcen. Obwohl dieser Artikel retrospektiv betrachtet sicherlich die

4 Solche Übersichten finden sich in Griliches (1978), Brockhoff (1992), Klodt (1995) und Stoneman (1995), um nur einige zu nennen. 
größte Aufmerksamkeit verdient, ist es verwunderlich, dass die anderen Beiträge fast gar nicht mehr zitiert werden. So gibt es weitere Beiträge zur formalen Modellierung von Forschung und Entwicklung, Überlegungen zu Nachfrage und Angebot erfinderischer Leistungen sowie zum Beitrag von unabhängigen Erfindern. Einige Verfasser (so Peck, Enos und Mueller) diskutieren Fallstudien, die zum damaligen Zeitpunkt die Forschungsarbeiten der RAND Corporation stark mitgeprägt hatten. ${ }^{5}$ Andere Arbeiten untersuchen, wie gut vorhersagbar Kosten, Zeitbedarf und Erfolgsaussichten von FuE-Projekten zum damaligen Zeitpunkt waren oder wie Entscheidungen in den untersuchten Projekten getroffen wurden.

Der Konferenzband spielte damals eine bedeutende Rolle, da wichtige Ökonomen sich hier eines Themas annahmen, das zuvor eher ein Schattendasein gefristet hatte. Zwar war die Bedeutung des gezielt herbeigeführten technischen Wandels als wichtige Quelle von Produktivitätswachstum schon früher in Arbeiten von Solow (1957) und anderen erkannt worden - die mikroökonomische Analyse hinkte zum damaligen Zeitpunkt aber hinterher. Arrows Beitrag - in dem die (nichtstrategischen) FuE-Anreize eines ex ante Monopolisten und eines ex ante im perfekten Wettbewerb stehenden Unternehmens mit denen eines sozialen Planers verglichen wurden - markierte die Hinwendung zu einer formalen Analyse der Erfindungstätigkeit. Weitere Meilensteine seien hier kurz erwähnt:

- Scherer (1967) untersuchte die Ressourcenallokation für FuE unter Rivalitätsbedingungen. Die von ihm verwendete Entscheidungsvariable ist nicht die Höhe der FuE-Aufwendungen, sondern der optimale Zeitpunkt der Technologieeinführung.

- Nordhaus (1969) studierte Eigenschaften des Patentsystems und betrachtet u.a. die Länge des Patentschutzes als Stellgröße für die Wirtschaftspolitik. Später knüpften anderer Verfasser wie Cornelli und Schankerman (1999) an diese Ergebnisse an und analysierten die Effizienzeigenschaften von Patentsystemen mit Verlängerungsgebühren.

- Loury (1979) formulierte ein Patentrennenmodell, in dem unter bestimmten Bedingungen - der Preis für den Gewinner des Patentrennens ist nicht von der Höhe der FuE-Aufwendungen beeinflusst - aus Sicht des sozialen Planers zuviel FuE stattfinden kann. Lee and Wilde (1980) verfeinerten dieses Modell.

5 Es ist bemerkenswert, dass die RAND-Forscher, unter ihnen Arrow, Nelson, Meckling und andere bekannte Ökonomen, ebenfalls vor dem Versuch einer allgemeinen Beschreibung des FuE-Prozesses zunächst etwa 50 Fallstudien, hauptsächlich im Bereich der Militärforschung, durchgeführt hatten. 
- Gilbert und Newbery (1982) stellten ein Modell vor, das die Persistenz von Monopolen nahelegt. Reinganum (1984) zeigte dahingegen, dass bei Unsicherheit bezüglich des Einführungszeitpunkts von Innovationen besonders drastische Neuerungen mit höherer Wahrscheinlichkeit von neu eintretenden Unternehmen als von im Markt etablierten Firmen kommen werden. ${ }^{6}$

- Spence (1984) legte eine theoretische Analyse von „Spillover-Effekten“ vor, die rein substitutiven Charakter in FuE-Aufwendungen haben. Er untersuchte in Simulationen, ob eine Stärkung der Eigentumsrechte über das Patentsystem oder eine Subventionierung von FuE sinnvoller sei.

- Cohen und Levinthal (1989) griffen psychologische Lernmodelle auf und untersuchten theoretisch und empirisch Spillover-Effekte, die von der Lernfähigkeit eines Unternehmens oder Individuums abhängig sind (absorptive Kapazität).

- D'Aspremont und Jacquemin (1988) formulierten ein Modell, in dem SpilloverEffekte komplementär sind (durch eigene und fremde FuE generierte Kostenreduzierungen also additiv auftreten). Dieses und andere Modelle dienten in der Folgezeit als Vehikel, um die volkswirtschaftlichen Auswirkungen von Research Joint Ventures theoretisch zu untersuchen.

- In der Literatur zum endogenen Wachstum von Ökonomien wurde in den 90er Jahren die Rolle von Forschungsanstrengungen, Spillover- und Lerneffekten im allgemeinen Gleichgewicht modelliert (vgl. Barro/Sala-i-Martin 1999).

- Ebenfalls in diesem Zeitraum wurde die Untersuchung des kumulativen bzw. sequenziellen Charakters des technischen Fortschritts weitergeführt, so in Arbeiten von Caballero/Jaffe (1993) und - bezogen auf das Patentsystem - von Green/Scotchmer (1995).

Nicht alle, aber erstaunlich viele der neueren Arbeiten können zumeist thematisch den Forschungsfragen zugeordnet werden, die bereits im von Nelson herausgegebenen Konferenzband diskutiert worden waren. Erstaunlich ist allerdings, dass Forschungsarbeiten zur Richtung der technischen Entwicklung - im NBERKonferenzband im Titel prominent vertreten und in etlichen Beiträgen diskutiert von nachfolgenden Verfassern in der Industrieökonomik fast kaum aufgegriffen

6 Dieses Resultat liefert eine rationale Erklärung für das in der betriebswirtschaftlichen Innovationsforschung häufig dokumentierte Phänomen, dass etablierte Unternehmen bei drastischem technischem Wandel häufig nicht an frühere Erfolge anknüpfen können. Vgl. Christensen 1993. Als alternative Erklärung gibt es aber auch die Vermutung, dass ein Organisationsversagen eine Rolle spielt. Vgl. hierzu auch den Beitrag von Daniels et al. in diesem Band. 
wurden, während das Thema in der Arbeitsmarktökonomik (,skill-capital complementarity“, „skill-biased technical change“), Umweltökonomik (,energy-saving technologies“) und Entwicklungsökonomik (,appropriate technology“) weiterhin große Aufmerksamkeit genoss. Erst seit den 80er Jahren sind wieder einige wenige industrieökonomische Arbeiten zu diesem Thema vorgelegt worden, beispielsweise von Cardon/Sasaki (1998) und Klette/de Meza (1986). In der Analyse von FuEBemühungen in der Biotechnologie spielt die Wahl der Forschungsrichtung ebenfalls wieder eine große Rolle (Harhoff et al. 2001). Sie ist auch forschungspolitisch relevant, wenngleich die Diskussion um die Instrumente der FuT-Politik meist auf die Frage zugeschnitten wird, ob in einer Marktwirtschaft ohne staatliche Eingriffe ausreichend viel FuE betrieben wird.

Ein wichtiger theoretischer Kontrapunkt zu der spieltheoretischen Literatur soll nicht unerwähnt bleiben. Nelson und Winter, die sich während der frühen Forschungsarbeiten zum Thema Innovation an der RAND Corporation kennengelernt hatten, stellten im Jahr 1982 mit ihrem Buch „An Evolutionary Theory of Economic Change“ eine Sicht des Innovationsprozesses vor, die als Alternative zu „orthodoxen" Modellen gedacht ist und den Eigenheiten des Innovationsprozesses Rechnung tragen soll. Nicht die Analyse von Gleichgewichten steht im Vordergrund, sondern die Dynamik von detailliert spezifizierten Sektoren, die per Simulationsrechnungen abgebildet wird.

Insgesamt ist festzustellen, dass die theoretische Analyse von Innovationsprozessen (wie andere Gebiete der Industrieökonomik) durch den Einsatz spieltheoretischer Ansätze eine Belebung erfahren hat. Gleichzeitig haben diese Modelle mit dem „embarrassment of riches“ zu kämpfen, mit der „peinlichen“ Vielfalt von Gleichgewichtssituationen, die sich theoretisch aufzeigen lassen. Die evolutorischen Modelle haben - trotz vieler wünschenswerter Eigenschaften - keinen Siegeszug in der ökonomischen Innovationsforschung antreten können. Letztlich generieren auch Simulationsrechnungen eine Vielzahl möglicher Aussagen.

Umso wichtiger scheint ein Blick auf einige wichtige Ergebnisse der empirischen Literatur zu sein - wiederum im Hinblick auf eine mögliche Beeinflussung der FuT-Politik. Für unsere Zwecke sind vier Bereiche der empirischen Literatur besonders interessant: Studien zu den Produktiviätsauswirkungen von FuE, Fallstudien zur Quantifizierung von privaten und sozialen Erträgen aus FuE, Arbeiten zur Quantifizierung von Spillovereffekten auf der Unternehmensebene und schließlich Studien zur Auswirkung von staatlicher Förderung auf die FuE-Anreize von Unternehmen. Auch hier sollen nur die wichtigsten Ergebnisse grob skizziert werden:

- Griliches (1998) fasst die Ergebnisse von zahlreichen Studien zusammen, die von ihm und anderen seit den 60er Jahren durchgeführt wurden. Diese Analysen zeigen, daß FuE einen deutlichen Beitrag zum Produktivitätswachstum liefert. 
- Mansfield und Koautoren (vgl. Mansfield et al. 1977) führten eine große Zahl von Fallstudien durch, in denen die privaten und sozialen Erträge von Innovationen gegenübergestellt wurden. Diese Studien zeigen durchweg, dass die sozialen Erträge erheblich über den privaten liegen. Das theoretisch mögliche Resultat, dass FuE exzessiv betrieben wird und so Wohlfahrtsverluste entstehen, ist empirisch nicht nachweisbar.

- Griliches (1994) faßt Forschungsergebnisse zur Existenz von Spillovereffekten zusammen. Solche Effekte lassen sich mit Unternehmens- und Industriedaten gleichermaßen nachweisen. Absorptionseffekte (entsprechend der Theorie von Cohen/Levinthal) sind ebenfalls empirisch nachweisbar. ${ }^{7}$

Wenn man diese empirischen Ergebnisse betrachtet - sowohl die Fallstudienevidenz von Mansfield und Koautoren als auch die ökonometrischen Ergebnisse von Griliches und anderen, dann können diese als empirische Belege für das Vorliegen eines Marktversagen interpretiert werden. Die Ergebnisse sind inzwischen für viele Länder repliziert worden, auch für Deutschland. Prima facie liegt also eine Berechtigung für staatliche Eingriffe vor. Allerdings ist damit immer noch nicht die Frage beantwortet, ob diese Eingriffe wirklich wohlfahrtserhöhend wirken.

Diese Frage hat die empirische Innovationsforschung seit langem beschäftigt. ${ }^{8}$ Von besonderem Interesse für unseren Beitrag sind Studien, die den Effekt von staatlichen Subventionen für private FuE-Anstrengungen zu quantifizieren versuchen. Führen staatliche Subventionen zu einem reinen „Crowding-out“ von privaten Anstrengungen, oder kann eine Komplementarität nachgewiesen werden? Nur im letztgenannten Fall macht es überhaupt Sinn, FuE-Fördermaßnahmen zu betreiben.

Die Beanwortung dieser Frage ist schwierig, da die real vorhandenen Daten nicht experimentellen Ursprungs sind, sondern bestenfalls als quasi-experimentell interpretiert werden können. Unternehmen, die sich um staatliche Förderung ihrer FuEProjekte bemühen, unterscheiden sich vermutlich erheblich von denen, die dies

7 Diese Ergebnisse haben vor einigen Jahren eine Debatte ausgelöst - wenn Spillover nur bei Vorliegen eigener FuE-Anstrengungen genutzt werden können, so in vereinfachender Darstellung die Position von Kealey (1997), dann liege keine Externalität mehr vor. Paul David (1997) zeigt in einer Diskussion, dass diese Auffassung nicht zutrifft. Vgl. auch Hoppe/Pfähler (2001).

8 Vgl. die Arbeiten von Adams und Jaffe (1996), Griliches (1998), Lichtenberg und Siegel (1991), Leyden/Link (1991), Levin/Reiss (1984), Mansfield et al. (1977) und Schmookler (1966). Für einen Überblick der bis 1994 erschienenen Literatur vgl. Stoneman (1995). 
nicht tun oder bei der Selektion der zu fördernden Projekte nicht erfolgreich sind. Selbst wenn also nachgewiesen wird, dass die FuE-Aufwendungen geförderter Unternehmen um mehr als die Förderbeträge über den FuE-Aufwendungen nicht geförderter Unternehmen liegen, so ist das Resultat u.U. gänzlich auf Selektionseffekte zurückzuführen.

Jüngere Fortschritte in der Wirkungsforschung haben wichtige Hinweise geliefert, wie trotz des Fehlens experimenteller Daten unter bestimmten Umständen solche Verzerrungen beseitigt werden können. Mit Hilfe ökonometrischer Methoden wird dabei (vgl. Lechner/Pfeiffer 2001) ein Vergleichssample von Individuen oder Unternehmen konstruiert, die dann direkt mit den „behandelten“ (in diesem Fall: geförderten) Einheiten verglichen werden können.

In den vergangenen Jahren sind eine Reihe von Studien entstanden, die zum Teil die neueren Korrekturverfahren einsetzen. Eine Übersicht wird von Klette et al. (2001) vorgestellt. In der u.E. ökonometrisch bisher am sorgfältigsten durchgeführten Studie kann Lach (2000) für israelische Unternehmen im Zeitraum 19901995 zeigen, dass pro DM staatlicher Förderung von den Unternehmen weitere 0,41 Geldeinheiten an zusätzlichen FuE-Mitteln eingesetzt werden - der FuEEinsatz erhöht sich also bei diesen Unternehmen insgesamt um 1,41 Geldeinheiten. Klette/Moen (1997) finden in einer Stichprobe norwegischer Unternehmen ebenfalls komplementäre Effekte, die aber wesentlich kleiner ausfallen als bei Lach. Andere Studien deuten darauf hin, dass der Fördereffekt sehr stark von der Auswahl der Unternehmen abhängt. Tovanen und Niininen (2000) warnen auf der Grundlage ihrer Ergebnisse davor, Fördermittel solchen Unternehmen zur Verfügung zu stellen, die entweder sehr hohen oder sehr niedrigen Cash-Flow haben. Die erstgenannten Firmen werden nach Meinung der Autoren nicht von der Subvention profitieren (Mitnahmeeffekte), während die letztgenannten keine Komplementäreffekte zeigen werden, da die eigenen Finanzmittel dafür nicht ausreichen. Für Deutschland liegen bisher Studien von Czarnitzki/Fier (2001) und Almus/Czarnitzki (2001) vor, in denen ebenfalls komplementäre Effekte gefunden werden. ${ }^{9}$

Derzeit ist es vermutlich zu früh, diese Studien abschließend zu bewerten. Aber die bisher vorliegenden Ergebnisse deuten - zumindest für Europa und Israel -

9 Czarnitzki/Fier (2001) untersuchen geförderte Firmen des Dienstleistungssektors. Die Ergebnisse ihrer OLS-Regressionen sowie eines Matching-Verfahrens zeigen, dass die direkte Projektförderung zu den privat finanzierten Innovationsaufwendungen komplementär ist. Almus/Czarnitzki (2001) widmen sich in ihrer Studie ostdeutschen Unternehmen. Der hier verwendete Matching-Ansatz zeigt ebenfalls, dass die Teilnahme an öffentlichen FuEFörderprogrammen komplementär ist und zu höheren privaten FuE-Intensitäten führt. 
nicht darauf hin, daß die Wirkung der staatlichen Förderung durch Mitnahme- oder „Crowding-out"-Effekte völlig aufgehoben wird. Aber dieses Ergebnis ist noch keine Absolution der staatlichen Förderung, da die Kosten der Projektadministration und andere Effekte hier nicht berücksichtigt sind. Erst eine volle KostenNutzen-Analyse würde es ermöglichen, über Sinn oder Unsinn von FuTFördermaßnahmen zu entscheiden.

\section{Forschungspolitik - klassische und neue Ansätze}

Lässt man die Forschungs- und Förderpolitik ${ }^{10}$ der vergangenen 50 Jahre Revue passieren, so lassen sich unterschiedliche Prioritäten, Instrumente und Förderschwerpunkte ausmachen. Eine direkte Anbindung an die oben skizzierten Fortschritte oder Strömungen der Innovationsforschung lässt sich dabei in den seltensten Fällen beobachten - gleichwohl hat die FuT-Politik immer wieder auf Diskurse in der Forschung reagiert, wenngleich mit langen Verzögerungen. Gerade beim Einsatz neuer Konzepte der Innovationsförderung gibt es derzeit aber auch Instrumente, deren ökonomische Konsequenzen wissenschaftlich bisher nicht sorgfältig durchdacht worden sind.

\section{Entstehungsgeschichte: FuT-Politik und Förderinstrumente}

Der Grundstein für die Forschungs- und Förderpolitik im Nachkriegsdeutschland wurde 1955 mit der Gründung des Bundesministeriums für Atomfragen gelegt. Die Etablierung des Ministeriums schaffte die institutionellen Voraussetzungen für eine systematische Wissenschafts- und Technologiepolitik, die das Ziel hatte, Gesetze zu entwerfen, internationale Abstimmungen wahrzunehmen und ein entsprechendes Förderinstrumentarium zu entwickeln. ${ }^{11}$ Die staatliche Förderung von FuE deckte sich weitgehend mit allgemeiner Wissenschaftsförderung und beschränkte sich auf Hochschulen und die Selbstverwaltungsorgane der Wissenschaft. Auf Grundlage

10 Wir beschränken uns in diesem Papier auf die Förderung von Forschung und Innovation durch das zuständige Bundesministerium. Weitere Maßnahmen der Länder und der EU lassen sich u.E. in die hier verwendeten Kategorien einordnen, bedürfen hinsichtlich ihrer Wirkungen aber einer separaten Analyse. Ebenso gingen und gehen von den Maßnahmen anderer Ressorts (z.B. des ehemaligen Postministeriums, des Gesundheitsministeriums u.a.) Wirkungen auf die Innovationsanreize privater Akteure aus, die wir hier aber nicht betrachten.

11 Nicht alle Nationalstaaten verfügen über ein entsprechendes Ressort - so flackert in den USA immer wieder eine Debatte auf, ob die Etablierung eines Ministeriums für Forschung und Technologie sinnvoll wäre. Bis heute existiert aber kein derartiges Ressort. 
dieser Organisation und ihrer Zielsetzungen traten weitere Aufgaben hinzu. Nachdem der Wirtschaft Starthilfen zur Durchführung von FuE in Großprojekten in der Atomforschung gewährt worden waren, dehnte sich diese Förderung in den sechziger Jahren auch auf andere Bereiche der Großforschung aus. Die Forschungspolitik war charakterisiert durch die institutionellen Förderung, die bis heute das Ziel verfolgt, größere Forschungseinrichtungen zu finanzieren, sowie von der direkten Förderung von Wissenschaft und Großindustrie. Mit der direkten Projektförderung finanziert der Bund ,punktuell“" FuE-Aktivitäten, die innerhalb von Fachprogrammen vergeben werden. Diese Fachprogramme haben als übergeordnete Themenbereiche zum Ziel, auf ausgewählten Technologiegebieten einen im internationalen Maßstab hohen Leistungsstand von FuE zu fördern. 1962 fasste der Bund den Beschluss zur planmäßigen Förderung der Weltraumforschung, an den sich 1967 das erste Fachprogramm zur Förderung der Datenverarbeitung anschloss. 1969 folgte die Meeresforschung und schließlich das breite Feld der sogenannten „Schlüsseltechnologien".

Ende der sechziger Jahre spitzte sich überdies die Diskussion um die technologische Rückständigkeit Europas gegenüber den Großmächten USA und UdSSR (,technology gap“) zu. Alarmiert durch die ausländischen Erfolge auf den Technologiegebieten Kernforschung, Weltraumfahrt und Datenverarbeitung war man sich einig, die Defizite in der Zukunfts- und Wettbewerbsfähigkeit Deutschlands durch ein verstärktes staatliches Engagement in der Forschungsförderung ausgleichen zu müssen. In den folgenden Jahren wurden die etablierten technikzentrierten Fördermaßnahmen zum einen um Programme ergänzt, die darauf abzielten, eine möglichst breite Palette an Forschungsthemen abzudecken. Zum anderen wurde der bisherige Zielkatalog um gesellschaftsbezogene Ziele und eine größere Verantwortung gegenüber der Umwelt erweitert. Die Forschungs- und Reformpolitik wurde zur zentralen politischen Aufgabe der siebziger Jahre (BMBW 1972: 20).

Die sozialliberale Koalition von 1969 hatte beschlossen, Forschung als Produktionsfaktor und Zukunftsvorsorge zu fördern und weitere Finanzmittel zur Verfügung zu stellen. Die FuT-Politik zielte darauf ab, ,besonders aufwendige und aussichtsreiche Sachgebiete der Forschung und Technologie vorrangig, konzentriert und koordiniert" (BMBW 1972: 93) zu fördern. Nach der bisherigen Phase der Imitation amerikanischer FuT-Politik war es nun das erklärte Ziel, eine ,effektivere, den gesellschaftlichen Bedürfnissen besser entsprechende forschungspolitische Zielsetzung“ (BMBW 1972: 11) zu finden. Die neue Bundesregierung unter Bundeskanzler Brandt wollte diesem Anspruch im Rahmen eines umfangreichen innenpolitischen Reformprogramms („mehr Demokratie wagen“) genügen und in diesem Sinne auch die Forschungspolitik neu ausrichten. In den siebziger Jahren wurden die immer wieder beklagten Lücken und Defizite in der deutschen Forschungslandschaft durch eine Reform- und Modernisierungseuphorie abgelöst, in 
deren Mittelpunkt der „Mensch und seine Umwelt“ standen (vgl. Krieger 1987: 263 f.).

Der Bund lenkte von nun an die Forschungsanstrengungen auch auf soziale Aspekte, um dem Menschen und seiner Umwelt besser gerecht zu werden (Daseins- und Vorsorgeforschung). In diesem Reformbestreben und mit der Neuorganisation des BMFT erhielt die FuT-Politik gleichzeitig eine stärker strukturpolitische Prägung. Diese Ausrichtung zielte primär darauf ab, die Forschungsförderung mit einer vom Staat anzustrebenden positiven gesamtwirtschaftlichen Entwicklung zu verknüpfen.

Unter dem Eindruck wirtschaftlicher Krisen sollte Forschungsförderung in der zweiten Hälfte der siebziger Jahre effizienter gestaltet und als Handlungsoption für die Forcierung des strukturellen Wandels eingesetzt werden. Im Mittelpunkt dieser strukturpolitischen Konzeption stand ausdrücklich die Stärkung leistungsfähiger Industriezweige durch eine selektive Förderung neuartiger Produkte und Technologien. ${ }^{12}$

Die strukturpolitische Diskussion um Schlüsseltechnologien, UnternehmensgröBen und Branchen erweiterte gleichzeitig den Adressatenkreis der direkten Projektförderung. Erste Studien aus den USA (Birch 1979) belegten anscheinend die volkswirtschaftliche Bedeutung kleiner und mittlerer Unternehmen (KMU), die in der Forschungspolitik und gegenüber der Großindustrie lange Zeit vernachlässigt worden waren. ${ }^{13}$ Der klassische Ansatz der direkten Projektförderung wurde von nun an auch auf KMU ausgeweitet. Im Laufe der Zeit musste jedoch festgestellt werden, dass die direkte Projektförderung - für die Großforschung konzipiert - für KMU wenig geeignet war. Das Antragsverfahren, die Erwartungen an die verfügbaren Kapazitäten sowie der Nachweis des gesellschaftlichen Nutzens eines Vorhabens übertrafen die Möglichkeiten kleinerer Unternehmen. Im Zusammenhang mit den wirtschaftlichen Zielgruppen und der Zweckbestimmung von Fördermitteln kam es Ende der siebziger Jahre zu einem Ressortkonflikt zwischen dem Forschungs- und dem Wirtschaftsministerium (BMFT und BMWi). Die hohen Förderquoten, die vielfältigen Förderfelder wie auch die zweifelhafte Selektion einzelner

12 Vgl. Hauff/Scharpf (1977, 39): „Die notwendige Konsequenz einer auf Stärkung der leistungsfähigsten Branchen und Unternehmen gerichteten staatlichen Steuer- und Förderungspolitik wird allerdings eine viel stärkere Spezialisierung unserer Industriestruktur sein. [...] Die Wahl und selektive Förderung dieser künftigen Wirtschaftsstruktur wird die eigentliche Aufgabe einer Strategie der aktiven Strukturpolitik sein.“

13 Die Studien von Birch und Koautoren hatten eine enorme politische Wirkung. Viele der von Birch damals gemachten Aussagen sind durch neuere Forschungsergebnisse widerlegt worden. Vgl. Davis et al. (1996). 
Unternehmen verzerrten aus ordnungspolitischer Sicht den Markt stärker, als dass sie ihm förderlich seien. Diese Debatte führte zur Entwicklung von anderen, eher breitenwirksamen Förderkonzepten. In Ergänzung zu projektorientierten Fördermaßnahmen wurde als weiteres Instrument die indirekt-spezifische Förderung mit dem Ziel eingeführt, lediglich Technikgebiete vorzugeben, um die Aufmerksamkeit von Unternehmen gezielter auf neue Produkt- und Verfahrensentwicklungen zu lenken.

In den achtziger Jahren sah die FuT-Politik nach der wirtschaftszentrierten Modernisierungsphase der siebziger Jahre ihre Aufgaben wieder stärker im vorwettbewerblichen Bereich. Nach 1982 kam es dem Subsidiaritätsprinzip entsprechend zu einer vermehrten „Zurückhaltung des Staates gegenüber der Forschung und Entwicklung in der Wirtschaft, insbesondere bei inhaltlichen Vorgaben“ (BMFT 1984: 5). FuT-Politik wollte ihre Aufgabe von nun an nicht mehr darin verstanden wissen, Art und Entwicklungsrichtung des technologischen Wandels direkt zu determinieren, sondern sah ihre Aufgabe in der Verbesserung der Rahmenbedingungen. In den Folgejahren führte der Bund sein Engagement, das primär immer noch der kerntechnischen Entwicklung galt, schrittweise zurück. Mit dem Aufbau von Versuchsreaktoren und der Einigung auf das Subsidiaritätsprinzip hatte die finanzielle Projektförderung an die Großindustrie ihren Höhepunkt erreicht. Von nun an sollte die Wettbewerbsfähigkeit der deutschen Wirtschaft durch Maßnahmen und Einrichtungen zur Förderung der Diffusion von Innovationen gestärkt werden. Beschleunigte Diffusionsprozesse sollten dazu beitragen, neue Technologien schneller in Produktionsprozesse einfließen zu lassen, um durch neue Produkte bzw. durch Produktivitätssteigerungen die volkswirtschaftliche Leistungsfähigkeit zu steigern. Die Bedeutung von Diffusionsprozessen wurde gerade in den Arbeiten von Ergas herausgestellt. Im internationalen Vergleich wurde der Bundesrepublik damals eine betont diffusionsorientierte Politik unterstellt. ${ }^{14}$

Forschungs- und Technologiepolitik war bis dahin von der Zielvorstellung geleitet, dass die Grundlagenforschung Potenziale schafft, die von Wirtschaft und Industrie erkannt, aufgegriffen und erfolgreich am Markt umgesetzt würden. In den nachfolgenden Phasen setzte eine qualitativ andere Förderstrategie neue Maßstäbe:

14 So differenziert Ergas (1987): „Technology policy in the United States, the United Kingdom, and France remains intimately linked to objectives of national sovereignty. Best described as 'mission-oriented', the technology policies of these nations focus on radical innovations needed to achieve clearly set out goals of national importance. In these countries, the provision of innovation-related public goods is only a secondary concern of technology policy. [...] In contrast, technology policy in Germany, Switzerland, and Sweden is primarlily "diffusionoriented" [...]“. 
Projektfördermittel wurden verstärkt über die Vergabeform der Verbundförderung eingesetzt. Der Bund definiert die Verbundforschung als ,arbeitsteilige Bearbeitung übergreifender, thematisch-funktionell zusammenhängender Problemstellungen in Forschung und Entwicklung durch mehrere Unternehmen und Forschungsinstitute" (BMFT 1984: 29). Die Vorstellung des automatischen Transfers von Ergebnissen der Grundlagenforschung in marktgängige Produkte wurde aufgegeben und stattdessen nach Möglichkeiten gesucht, wie sich „Wissen“ aus der Wissenschaft in die Wirtschaft transferieren lässt. Verbundprojekte waren zunächst ein Instrument zur Initiierung einer zielgerichteten multidisziplinären Zusammenarbeit zwischen verschiedenen Forschungseinrichtungen, wie z.B. Universitäten, Großforschungseinrichtungen und Max-Planck-Instituten, später aber auch zwischen diesen und der Industrie. Seit den achtziger Jahren ging diese ,prozedurale Neuerung" vor allem in die direkte Projektförderung ein, so dass heute zahlreiche der direkt geförderten FuE-Projekte Verbundprojekte sind. Als Vorteil gegenüber der einzelunternehmerischen Projektförderung wird angeführt, dass sich durch die Bildung von Forschungsverbünden vorhandene und sich gegenseitig ergänzende personelle und infrastrukturelle Ressourcen mehrerer Disziplinen zur Bearbeitung eines bestimmten Problems nutzen lassen. Dieser Gedanke des Wissens- und Technologietransfers durch Kooperationen zwischen Wissenschaft und Praxis prägt die Forschungsförderpolitik bis heute. Unter dem Schlagwort „Wissenstransfer über Köpfe" wird beispielsweise der Personalaustausch zwischen Forschungsinstitutionen und Unternehmen gefördert.

In den neunziger Jahren wurde dieser Kooperationsgedanke weiter ausgebaut. Nach der dirigistischen Phase von FuT-Politik Ende der siebziger Jahre ging es nun darum, die Förderung auf eine leistungsorientierte Mittelvergabe in Kooperationen und Wettbewerben umzustellen. FuT-Politik zielte im Besonderen darauf ab, Eigenverantwortung und Selbständigkeit zu stärken. Der Bund griff in dieser Zeit die Diskussion um nationale Innovationssysteme auf und konzipierte eine Förderpolitik, in der der Staat moderierend zur Stimulierung von Netzwerken beisteuert. Im Rahmen der Projektförderung wurden Leitprojekte initiiert, die auf „bahnbrechende wirtschaftlich verwertbare Produkt- und Prozessinnovationen abzielten“ (Rüttgers 1996: 295). Leitprojekte entspringen dem Kooperationsgedanken der Verbundförderung. Sie sind disziplinen- und branchenübergreifend als Ideenwettbewerbe angelegt und sollen marktfähige Innovationen auf strategischen, für die Volkswirtschaft bedeutsamen Feldern fördern.

Etwa zur gleichen Zeit setzt sich eine „regionenorientierte Innovationspolitik“ (Dohse 2000) durch, die als zentrale Elemente die Stimulierung des Wettbewerbs zwischen Regionen sowie die Förderung regionaler Cluster zum Gegenstand hat. Seither gilt es nicht nur, Unternehmen und Forschungseinrichtungen miteinander zu vernetzen, sondern die Stärken ganzer Regionen zu bündeln. Diese Entwicklung 
greift die Beobachtung der Innovationsforschung auf, dass private FuE, Gründungen im Hochtechnologiebereich und universitäre Forschung in großem Ausmaß in Agglomerationen ablaufen. Prototypisch sind die Wettbewerbe „BioRegio“ und „InnoRegio“ zu nennen. Zum einen ist beabsichtigt, durch die Konkurrenz um erfolgversprechende Innovationskonzepte die Kooperationsneigung weiter auszudehnen, weil auch weniger bevorteilte Netzwerkpartner (Kammern, Verbände, Institute) einbezogen werden. Zum anderen soll das regionale Forschungsprofil geschärft werden - komparative Vorteile einer Region gegenüber anderen Regionen sollen stärker betont werden.

In neueren Ansätzen rückt auch die Innovationskompetenz mittelständischer Unternehmen wieder stärker in den Vordergrund. Mit den Förderprogrammen „ProInno“ und „InnoNet“ werden Kooperationen bzw. Verbundprojekte bezuschusst, in denen Forschungseinrichtungen und KMU trägerübergreifend ${ }^{15}$ zusammenarbeiten.

Der Netzwerkgedanke, dem zufolge alle Teilnehmer des Innovationssystems zur Kooperation zu motivieren seien, setzte sich auch in themenspezifischen Wettbewerben mit der Tendenz fort, Kompetenzzentren zu identifizieren, um diese im weltweiten Maßstab zu profilieren. In der Absicht, im internationalen Standortwettbewerb wieder mehr Profil zu gewinnen und die Stärken der heterogen gewachsenen deutschen Forschungslandschaft transparenter $\mathrm{zu}$ machen, werden Ideenwettbewerbe ausgeschrieben und Initiativen gestartet, die der Bund moderiert und fördert. Flankiert wurde diese Zielsetzung durch Vorhaben zur Verbesserung der Forschungsstruktur, Verbesserung des unternehmerischen Zugangs zu Beteiligungskapital und durch Deregulierung. Trotz dieser neuen Systemansätze treten die herkömmlichen Förderinstrumente nicht in den Hintergrund. Vielmehr soll die breite Palette unterschiedlicher Vergabeformen öffentlicher Mittel die bestehenden Maßnahmen zur Förderung von FuE-Aktivitäten ergänzen. Die Forschungsförderung existiert im 21. Jahrhundert weiterhin als Einzel- und Verbundförderung, wobei die Selektion der Fördermittelempfänger durch Leitprojekte wie durch die Netzwerkeförderung in Wettbewerben eine neue Dimension erhalten hat.

Letztlich sollte die Evolution der Förderung von technologieorientierten Unternehmensgründungen hier nicht unerwähnt bleiben. ${ }^{16}$ Noch zu Anfang der 90er Jahre war es nicht ungewöhnlich, dass die volkswirtschaftliche Bedeutung von tech-

15 Trägerübergreifende Maßnahmen erfordern die Beteiligung verschiedener Forschungseinrichtungen in einem Forschungsprojekt.

16 Die Bedeutung dieser Unternehmen, insbesondere die Gründung von Unternehmen im Umfeld von Universitäten, wird von Meyer-Krahmer und Kulicke in diesem Band detailliert untersucht. Die bei schnellwachsenden Unternehmen häufig zu beobachtende Venture-CapitalFinanzierung ist Thema der Analyse von Gebhardt/Schmidt in diesem Band. 
nologieorientierten Gründungen von der deutschen Wirtschaftspolitik, aber auch von Vertretern etablierter Unternehmen in Frage gestellt wurde. Angesichts des rasanten Wachstums von NASDAQ-notierten US-amerikanischen Gründungen verloren diese Stimmen an Bedeutung. Das Fehlen geeigneter Finanzierungsmöglichkeiten für Hochtechnologiegründungen ließ sich ebenfalls nicht mehr leugnen. $\mathrm{Zu}$ den ersten Maßnahmen des Bundes hatte die Finanzierung der Deutschen Wagnisfinanzierungs-Gesellschaft mbH (WFG) gehört, die bereits in den siebziger Jahren darauf ausgerichtet war, neu gegründeten bzw. jungen Technologieunternehmen Kapital für risikoreiche FuE-Vorhaben zur Verfügung zu stellen. ${ }^{17}$ Ab 1983 wurden technologieorientierte Unternehmensgründungen (TOU) in Form von Zuschüssen zu deren FuE-Aufwendungen sowie über Bürgschaften gefördert. Mit dem Modellversuch TOU (Förderung technologieorientierter Unternehmensgründungen) sollte die Produktionsvorbereitung und der Markteinstieg junger Unternehmen erleichtert werden. Der anschließende Modellversuch „Beteiligungskapital für junge Technologieunternehmen (BJTU)“ zielte ab 1989 darauf ab, Beteiligungskapitalgesellschaften zur Finanzierung von High-Tech Start-ups zu motivieren. Hierbei handelt es sich um einen „radikalen Instrumentenwechsel“" von der Zuschuss- zur Beteiligungsförderung (s. Abbildung 1), der bis heute mit dem Förderprogramm „Beteiligungskapital für junge Technologieunternehmen (BTU)“ praktiziert wird. ${ }^{18}$ Nicht unbeteiligt an diesem Wechsel war die in vielen Studien der jüngeren Innovationsforschung betonte Erkenntnis, dass eine Fremdkapitalfinanzierung klassischen $\mathrm{Mu}$ sters für Jungunternehmen, die nur über intangibles Kapital in Form von Wissen oder Geschäftsideen verfügen, nicht sinnvoll ist. ${ }^{19}$

17 Vgl. Mayer/Müller (1991).

18 Vgl. Kulicke/Wupperfeld (1996) und Lessat et al. (1999).

19 Auch hierzu sei auf den Beitrag von Gebhardt/Schmidt in diesem Band verwiesen. 
Abbildung 1: Etappen der Forschungs- und Innovationspolitik

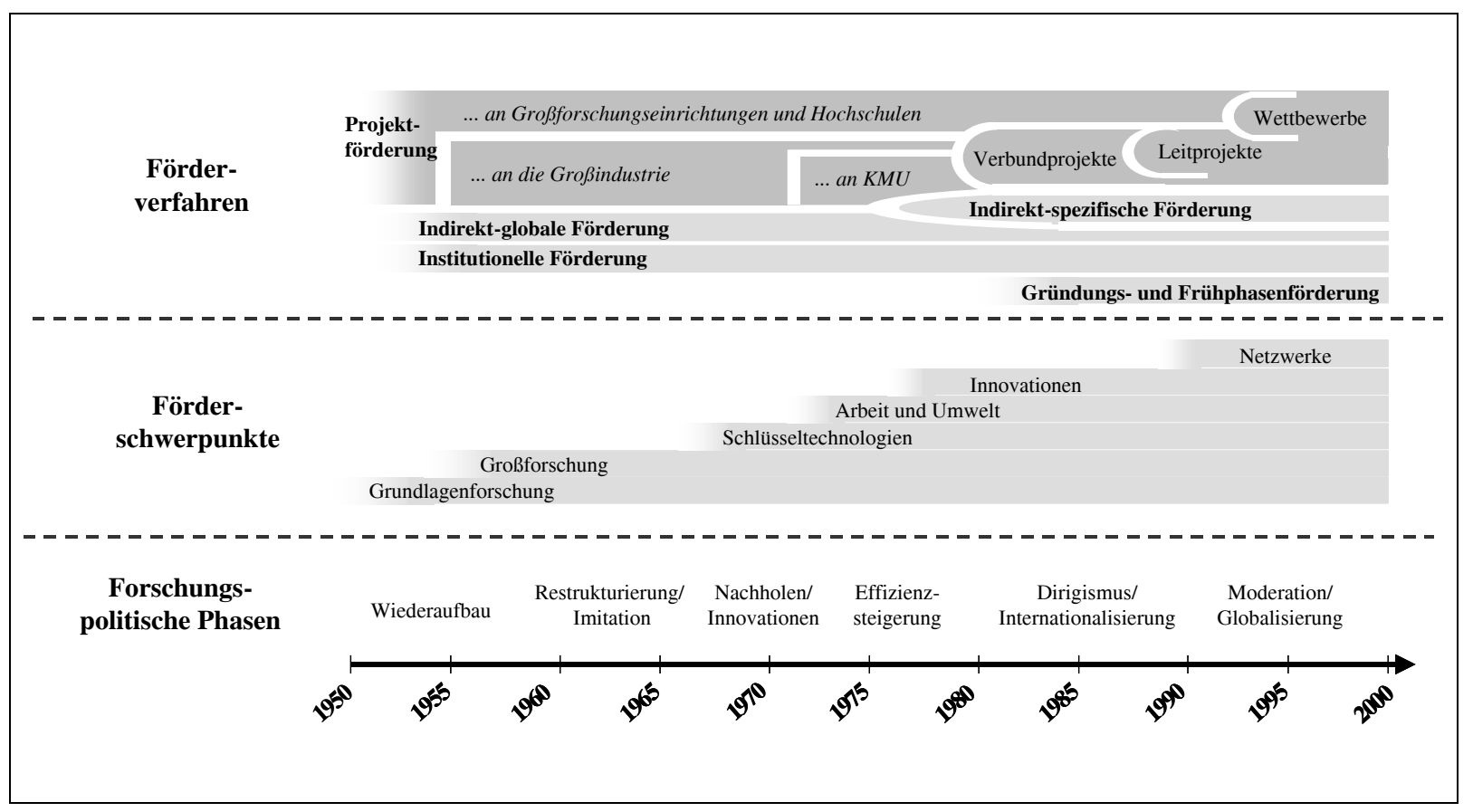

Klassische und neue Ansätze der Forschungspolitik - Potenziale und Gefahren

Forschungsförderung wird im Allgemeinen von der Wirtschaftspolitik in der Hoffnung akzeptiert, dass sie Initial- und Multiplikatoreffekte erzeugt, die zu besseren Marktergebnissen führen. Um ordnungspolitischen Bedenken Rechnung zu tragen, hat sich die Forschungsförderung in den vergangenen Jahrzehnten im Hinblick auf ihre Zielgruppen und Vergabeverfahren immer weiter differenziert. Wurden in den fünfziger Jahren nur einzelne Großforschungseinrichtungen und Universitäten gefördert, erweiterte sich der Kreis der Zuwendungsempfänger zunächst um die Großindustrie und später auch um mittelständische Firmen. Über den Netzwerkansatz der neunziger Jahre wurden schließlich alle Akteure des Innovationssystems in Projektskizzen und Förderverfahren potenziell einbezogen. Die klassische Forschungs- und Wissenschaftsförderung hat sich im Hinblick auf ihre Klientel und ihre Vergabeformen zu einer umfassenden Innovationsförderung gewandelt. Damit einhergegangen ist allerdings auch eine Zunahme der Zahl der Förderprogramme die Komplexität des Fördersystems ist selbst zu einer Gefahr für dessen ökonomische Effizienz geworden (Harhoff/Röller 1999, 487).

In der zeitlichen Abfolge wird das Bestreben der FuT-Politik deutlich, die jeweiligen vermuteten Defizite anderer Steuerungsformen zu kompensieren - ohne diese vollständig abzulösen. Heute entfallen rund 44 Prozent aller FuE-Ausgaben des Bundes (16 Mrd. DM) auf die institutionelle Förderung, 38 Prozent auf die direkte Förderung, 14 Prozent auf Hochschulen und die internationale Zusammenarbeit 
und vier Prozent auf die indirekte Förderung. Dabei ist die Ausgestaltung der Forschungsförderung deutlich komplexer geworden. Neben der klassischen Förderung einzelner Großprojekte und Industrien wird durch Förderwettbewerbe eine Konkurrenzsituation zwischen allen Forschungsinteressierten geschaffen, die zu Kooperationen, gemeinsamen Konzepten und zu Spitzenleistungen anregt. Diese neuen Vergabeformen werden vielfach als „,neuer Systemansatz“ bezeichnet. Worin unterscheiden sich diese neuen Ansätze vom klassischen Förderinstrumentarium?

Der Bund will mit Wettbewerben, bei Beibehaltung seiner ordnungspolitischen Funktionsleistungen, im Wesentlichen drei Ziele erreichen:

- Zum einen wird die häufig kritisierte, einseitige staatliche Festlegung von detaillierten Förderinhalten ersetzt durch eine übergeordnete thematische Vorgabe, die dann aber durch die FuE-Akteure selbst weiter spezifiziert wird. Zum anderen verspricht man sich durch Wettbewerbe um öffentliche Mittel mehr Dynamik bei der Ideenfindung und Umsetzung. Im Unterschied zu klassischen Förderverfahren sollen außerdem Akteure angesprochen werden, die bisher noch nicht in den Innovationsdialog eingebunden waren. Zusammenwirken sollen möglichst viele Partner aus Wirtschaft, Bildung und Forschung, Verwaltung sowie weitere Institutionen und Personen, die sich bei der Skizzierung und Umsetzung von Innovationskonzepten engagieren wollen (vgl. BMBF/BMWI 2000: 32).

- Zweitens haben die neuen Systemansätze aus Sicht des Fiskus den entscheidenden Planungsvorteil, dass im Unterschied zur fachprogrammbezogenen Projektförderung ein fixes FuE-Budget als „Preisgeld“ (Obergrenze staatlicher Ausgaben) vorgegeben wird. In der Qualifizierungsphase der Wettbewerbe wird frühzeitig der Kreis der Adressaten festgestellt, der in der anschließenden Entwicklungsphase Konzepte entwickelt, die dann nach einer Jury-Entscheidung in der Umsetzungsphase gefördert werden. Dagegen steht bei mehrjährigen Fachprogrammen nicht fest, ob zu einem frühen oder späteren Zeitpunkt besonders viele oder wenige Förderanträge gestellt werden. Der Bund muss somit nicht mit einer plötzlichen, die Haushaltsansätze sprengenden, sehr hohen Anzahl an Projektanträgen und dem damit verbundenen Mittelabfluss rechnen.

- Drittens erweitert der Bund - trotz stagnierender Haushaltsmittel - seine forschungspolitische Reichweite. Obwohl Wissenschaft und Großindustrie weiterhin zu den bevorzugten Adressaten der Forschungsförderung zählen, werden immer mehr kleine und mittlere Zuwendungsempfänger, Verbände u.a. in die Förderung einbezogen. Die Zahl der Zuwendungsempfänger hat sich in den neunziger Jahren nahezu verdoppelt. Der Bund finanziert inzwischen jährlich über 3.500 Fördermittelempfänger in mehr als 12.000 Vorhaben, die mittelbar oder unmittelbar auf FuE ausgerichtet sind. 
Kritisch lässt sich gegen diese Wettbewerbselemente anführen, dass eine ausgleichsorientierte Förderpolitik nicht mehr gewährleistet ist. Die Wettbewerbskriterien sind zweifelhaft und es handelt sich lediglich um eine Politik des ,picking the winners". Gerade die Vorteile der heterogenen deutschen Forschungslandschaft mit ihren interdisziplinären Netzwerken, so die Befürchtung, stehe durch in Wettbewerben herbeigeführte Abgrenzungsstrategien zur Disposition. Die Verlierer eines Wettbewerbs würden von Forschungsentwicklungen finanziell abgekoppelt und könnten auch diejenigen regionalen Akteure nicht mehr für sich gewinnen, die vor der Entscheidung der Wettbewerbsjury positiv eingestellt waren. Der Wettbewerb als Preisausschreiben benachteilige demnach die Verlierer doppelt: zum einen finanziell, zum anderen durch ein staatlich zertifiziertes Versagen (mutmaßliches Fehlkonzept). Unter diesen Begleiterscheinungen seien Wohlfahrtsverbesserungen durch Wettbewerbe fraglich. Es zeichnet sich aber auch das Risiko ab, dass der Bund seinen Verwaltungsapparat mit diesen Aufgaben überfordert: Immer mehr Unternehmen und Kooperationspartner erfordern die Aufmerksamkeit der Förderadministration. Die Moderation und Betreuung technologieübergreifender Netzwerke gestaltet sich jedoch ungleich aufwendiger und anspruchsvoller als die traditionelle Förderung einzelner Unternehmen. In der Konsequenz muss FuTPolitik selbst flexibel und innovativ sein. Der gegenwärtige Mix aus Einzel-, Verbund- und Wettbewerbsförderung ist insofern auch als Versuch zu werten, in der Forschungspolitik neue Wege zu gehen.

\section{Wissenschaftliche Fundierung und Evaluation der FuT-Politik}

Vor dem Hintergrund der jüngeren Entwicklungen in der Innovationsförderung stellt sich die Frage nach ihren Auswirkungen: Wie effizient ist die Forschungsförderung angesichts des breiten Spektrums an Instrumenten und Einsatzmöglichkeiten? Gelingt es dem Bund überhaupt, mit seinen Fördermaßnahmen zusätzliche FuE-Investitionen zu stimulieren oder überwiegen Mitnahmeeffekte? Sollte auf die ,wettbewerbsorientierte“ Förderung ganz verzichtet werden - zugunsten einer stärkeren Förderung der Grundlagenforschung? Bei der Beantwortung dieser Fragen gibt es - insbesondere für die FuT-Politik in Deutschland - immer noch erheblichen Nachholbedarf. Antworten auf diese Fragen sind erforderlich, um ordnungspolitisch oder anderweitig begründete Bedenken gegen eine Verstärkung der Marktnähe der Forschungsförderung auszuräumen - oder um die Weichen in der FuT-Politik neu stellen zu können. Die Idealvorstellung für solche Evaluationen ist dabei einfach beschrieben: Sie sollten eine Quantifizierung der Auswirkungen von FuT-Fördermaßnahmen in der Form von Kosten-Nutzen-Analysen umfassen, 
die den sozialen Ertrag der Fördermaßnahmen abschätzen. Außerdem sollten qualitative Förderwirkungen ausgewiesen werden, die nur schwer quantifizierbar sind. Unserer Ansicht nach wird der zweite Aspekt dieses Ideals sehr wohl in bundesdeutschen Evaluationen abgedeckt, während die quantitative Komponente nach wie vor massiv vernachlässigt wird. ${ }^{20}$

Quantitative Methoden der Wirkungsforschung zielen letztlich darauf ab, die Wirkungen von Politikmaßnahmen in Geldeinheiten zu messen, um eine KostenNutzen-Analyse zu ermöglichen. Zum Einsatz kommen hier standardisierte Fragebögen, szientometrische und bibliometrische Analysen ${ }^{21}$ sowie neuere ökonometrische Verfahren, bei denen den geförderten Unternehmen oder Institutionen eine Vergleichsgruppe gegenübergestellt wird. Gerade in der Forschungsförderung ist es allerdings problematisch, für eine Gruppe geförderter Unternehmen eine passende Kontrollgruppe zu finden, die die entsprechende Fördermaßnahme nicht in Anspruch genommen hat, ansonsten aber identische Ausgangsvoraussetzungen mitbringt. Sogenannte stochastische Matching-Verfahren werden aber in der neueren Literatur (insbesondere in der Arbeitsmarktökonomik) erfolgreich eingesetzt und stellen eine wichtige Ergänzung des Evaluationsinstrumentariums dar. ${ }^{22}$

Der Nachteil dieser Methoden liegt darin, dass durch die notwendige Abstraktion und Standardisierung der Datenerfassung Informationen verloren gehen, die für eine Analyse wichtig sein können. Der Einsatz ökonometrischer Verfahren bietet die Möglichkeit, die Auswirkungen von politischen Maßnahmen unter gleichzeitiger Berücksichtigung anderer Einflussfaktoren auf mikro- oder makroökonomischer Ebene zu analysieren. Diese Verfahren erfordern jedoch - insbesondere bei komplexen Schätzmodellen - Zugriff auf ausreichend hohe Fallzahlen.

Aus diesen Gründen betrachten wir qualitative Methoden ${ }^{23}$ als das natürliche Komplement zu quantitativen Verfahren, nicht jedoch als Ersatz. Diese Verfahren haben den Vorteil, dass sie für eine Analyse auch sehr komplexer Sachverhalte und Probleme eingesetzt werden können. So werden sie zur Beurteilung wissenschaftli-

20 Ein exzellentes Beispiel für die gelungene Kombination dieser Element stellt eine Studie des australischen Bureau of Industry Economics dar, in der die Wirkung von steuerlichen Vergünstigungen für FuE in Australien untersucht wurde (BIE 1993). In zahlreichen Ländern wird inzwischen eine Kosten-Nutzen-Analyse bereits bei Beginn von Fördermaßnahmen fest eingeplant.

21 Szientometrische und bibliometrische Verfahren analysieren z.B. Patentanmeldungen und Publikationen.

22 Vgl. hierzu die Ausführungen in Abschnitt 2 sowie Lechner/Pfeiffer (2001).

23 Vgl. hierzu Kuhlmann/Holland (1995). 
cher Fachkompetenz, zur Projektauswahl, Technikvorausschau, frühen Abschätzung gesellschaftlicher Konsequenzen und umweltbezogener Folgen eingesetzt. Der Vorteil von Fallstudien und Expertenbefragungen liegt in ihrem hohen Detaillierungsgrad. Allerdings bergen Befragungen von geförderten Unternehmen die Gefahr, dass die Befragten zu strategischen Antworten neigen, weil sie sich z.B. den Erfolg eines Förderprogramms wünschen oder weil sie sich Chancen für eine Anschlussförderung ausrechnen. Alternativ werden auch Interviews mit den Verantwortlichen der staatlichen Forschungsförderprogramme oder ihrer Projektträger geführt. ${ }^{24}$ Weitere Nachteile qualitativer Verfahren liegen in den hohen Kosten von Befragungen sowie in Selektionsproblemen bei der Bestimmung der Zielgruppe. Auch ist es denkbar, dass Daten durch Evaluatoren verzerrt werden, die im Falle allzu negativer Resultate um ihre eigenen Aufträge fürchten (vgl. Capron 1992, Luukonen 1998).

Gerade die „neuen Systemansätze“ der Forschungsförderung in Deutschland erfordern neue Vorgehensweisen in der Wirkungsanalyse. Wir skizzieren im Folgenden einige der Probleme, die hier zu lösen sein werden:

\section{Verwendung moderner Evaluationsverfahren}

Um Aussagen zur gesamtwirtschaftlichen Wirkung von FuE-Fördermitteln treffen zu können, sollen zunehmend moderne quantitative Verfahren eingesetzt werden. Diese Forderung stößt sowohl bei denen, die die Maßnahmen administrativ betreuen, als auch bei den Geförderten nicht immer auf Zustimmung. Widerstände in der Wirtschaftspolitik können mit „career concerns“ erklärt werden, mit der Befürchtung also, dass ein schlechtes Ergebnis eines Förderprogramms für die Karriere des Administrators schädlich ist. Die geförderten Unternehmen fürchten u.U. um die Fortsetzung von Programmen, in denen hohe Mitnahmeeffekte nachgewiesen werden können. Die besondere Brisanz von quantitativen Aussagen liegt darin, dass diese Ergebnisse nicht wie qualitative Äußerungen relativ leicht „wegdiskutiert“ werden können. Dem steht - aus gesellschaftlicher und politischer Sicht - der Gewinn an Informationen entgegen, der aus systematisch durchgeführten KostenNutzen-Analysen erwachsen könnte. Die hierfür erforderlichen Methoden der mikrökonomischen Wirkungsforschung werden in Deutschland im Unterschied zu anglo-amerikanischen Ländern noch sehr wenig angewendet. Kontrollgruppenansätze sowie die Berücksichtigung von Endogenitäts- und Selektivitätsproblemen bleiben in den meisten Evaluationen unbeachtet.

24 Ein Literaturüberblick findet sich bei Mansfield (1996). 


\section{Evaluationsqualität und Interessenkonflikte}

Die Evaluation komplexer Innovations- und Förderprozesse ist zeitintensiv. Um aussagekräftige Ergebnisse erzielen zu können, setzt die Befragung von Entscheidungsträgern in Forschungseinrichtungen und Unternehmen erfahrene Interviewer und kompetente Gesprächspartner voraus. Da der Markt für solche Experten in Deutschland nicht dicht besetzt ist, wird es zwischen Evaluatoren und Administratoren immer wieder zu wiederholten Interaktionen kommen - dies kann die Neutralität der Evaluation wieder bedrohen. Angesichts der rapiden technologischen Entwicklung, z.B. in den Informations- und Kommunikationstechnologien sowie in der Bio- oder der Nanotechnologie, sind zeitraubende Methoden der Wirkungsforschung nicht immer mit den Vorstellungen der FuT-Politik vereinbar. In einigen Ländern wird daher inzwischen auf begleitende Evaluationen (monitoring) übergegangen, die nicht nur ex post die Wirkung der FuE-Förderung untersuchen, sondern schon während der Förderphase Ergebnisse liefern. Sofern solche Anpassungen zu einer volkswirtschaftlichen Verbesserung führen, sind sie zu begrüßen. Allerdings besteht hier natürlich auch die Gefahr der Instrumentalisierung der Evaluation dann jedenfalls, wenn die Ergebnisse der Wirkungsforschung eingesetzt werden, um die Zielsetzung der Fördermaßnahmen nachträglich an die realisierten Ergebnisse anzupassen.

\section{Evaluation von Wettbewerben und Netzwerken}

Die Evaluation von Wettbewerben und Netzwerken steckt noch in den Kinderschuhen. Dies ist zum einen darauf zurückzuführen, dass diese Förderverfahren erst in den letzten Jahren zum Einsatz kamen und die ersten Wettbewerbe bis heute noch nicht abgeschlossen sind. Zum anderen gibt es noch keine Erfahrung und nur wenige Konzeptionen, wie das staatliche Engagement bei diesen Kooperationsformen zu analysieren ist. Woher nimmt der Bund also die Vorstellung, dass Wettbewerbe die „bessere“ Form der Innovationsförderung darstellen? Aus theoretischer Sicht ließen sich durchaus Gründe für ein solches Vorgehen anführen, wenn bspw. die marginalen Effekte der Förderung in den Gewinnerregionen erheblich über denen der anderen Regionen liegen oder wenn die Anstrengungen der Regionen im Wettbewerb selbst bereits positive gesamtwirtschaftliche Auswirkungen haben. Unseres Wissens gibt es dafür noch keine Belege, auch sind die zu berücksichtigenden Effekte in einer theoretischen Modellvorstellung bisher nicht beleuchtet worden. Die Beurteilung der Regionalwettbewerbe ist aber keineswegs einhellig positiv. Beispielsweise wird beim BioRegio-Wettbewerb von Kritikern die These vertreten, dass im Wettbewerb unterlegene Biotechnologie-Regionen diskriminiert werden. Ein wissenschaftlich empirischer Beleg für diese These steht bis heute aus, leitende Ministerialbeamte weisen vielmehr darauf hin, dass es gerade bei den Verlierern unter den Wettbewerbsteilnehmern durchaus positive Entwicklungen gebe. Der verschärfte Wettbewerb und "best practice"-Beispiele, so die Argumentation, 
führe bei den unterlegenen Wettbewerbsteilnehmern zu verstärkten Anstrengungen, um ihre Stärken - auch ohne staatliche Fördermittel - unter Beweis zu stellen. Aber auch dazu fehlt bisher jegliche Form systematisch erhobener empirischer Evidenz.

\section{Prinzipielle Wahl der Förderinstrumente}

Die Bundesregierung sieht zur Projektförderung keine Alternative. Für sie ist diese Maßnahme wichtig, ,weil mit diesem Instrument sehr flexibel auf neue Herausforderungen reagiert werden kann, sie in besonderer Weise geeignet ist, die Kooperation zwischen Forschung und Wirtschaft zu initiieren sowie durch ihren Wettbewerbscharakter Qualität zu fördern“ (BMBF 2000: 16). Aus wissenschaftlicher Sicht ist dem entgegenzuhalten, dass ein flexibler Staat auch immer wieder in Versuchung geführt wird, Lobby-Bestrebungen in einzelnen Bereichen kurzfristig nachzugeben. Gerade um diese Effekte zu vermeiden, bevorzugen andere Länder die steuerliche Begünstigung FuE-treibender Unternehmen als eine weniger selektive und wettbewerbsverzerrende Alternative. Auch in der bundesdeutschen Debatte wird sporadisch über solche Förderansätze diskutiert. Der Trade-off dieser beiden Instrumente liegt in Nachteilen durch vermeintlich hohe Mitnahmeeffekte bei steuerlichen Erleichterungen gegenüber Nachteilen der Selektion von Fördermittelempfängern in der direkten Projektförderung. Solange aber für die von der Bundesregierung bevorzugte Projektförderung keine aussagekräftigen Daten zur Effizienz der Mittelverwendung vorliegen, wird diese Debatte ein Wortgeplänkel bleiben.

Angesichts von mehr als 33 Milliarden DM, die Bund und Länder jährlich zur Förderung von Wissenschaft und Forschung aufbringen, erscheint die wissenschaftliche Auseinandersetzung mit der Wirkung dieser Gelder zwingend geboten. Es ist wohl keine Zumutung, von der FuT-Politik zu fordern, dass die von ihr initiierten Maßnahmen wissenschaftlich zu evaluieren sind. Da nur die Programmadministratoren über die geeigneten Daten verfügen, sind sie in der Lage solche Evaluationen entweder zu ermöglichen oder zu blockieren. $\mathrm{Zu}$ wünschen wäre aber auch, dass die empirische Wirtschaftsforschung in Universitäten und Instituten sich stärker auf diese Themen einläßt. 


\section{Literaturverzeichnis}

Adams, J. D. und A. B. Jaffe (1996), Bounding the effects of R\&D: An investigation using matched established-firm data, Cambridge (MA).

Almus, M. und D. Czarnitzki (2001), „The Effects of Public R\&D Subsidies of Firms“ Innovation Activities in a Transition Economy: The Case of Eastern Germany,“ ZEW Discussion Paper 01-10, Mannheim.

Barro, R. J. und X. Sala-i-Martin (1999), Economic Growth, Cambridge, Mass.

BMBF (2000), Bundesbericht Forschung 2000, Bundesministerium für Bildung und Forschung, Bonn.

BMWi (2001), „Wirtschaftliche Förderung - Hilfen für Investitionen und Innovationen,“ Juli 2001.

BMWi/BMBF (2000), „Innovationsförderung - Hilfen für Forschung und Entwicklung,“ Juli 2000.

BMBF/BMWI (2000), Innovationsförderung - Hilfen für Forschung und Entwicklung, Bundesministerium für Bildung und Forschung \& Bundesministerium für Wirtschaft und Technologie, Bonn.

BMBW (1972), Forschungsbericht IV der Bundesregierung, Bundesministerium für Bildung und Wissenschaft, Bonn.

BMFT (1984), Bundesbericht Forschung 1984, Bundesministerium für Forschung und Technologie, Bonn.

BIE (1993), R\&D, Innovation and Competitiveness: An Evaluation of the Research and Development Tax Concession. Canberra: Australian Government Publishing Service.

Birch, D.L. (1979), The Role of Small Business in New England, Cambridge MA.

Caballero, R. J. und A. B. Jaffe (1993), „How High Are the Giants' Shoulders: An Empirical Assessment of Knowledge Spillovers and Creative Destruction in a Model of Economic Growth," in: O. J. Blanchard und S. Fischer(Hrsg.), NBER Macroeconomics Annual 1993. Cambridge and London: MIT Press, 15-74.

Capron, H. (1992), Economic Quantitative Methods for the Evaluation of the Impact of R\&D Programmes: A State of The Art Monitor Spear, Komission der Europäischen Gemeinschaft, Luxembourg.

Cardon, J.H. und D. Sasaki (1998), „Preemptive search and R\&D clustering,“ RAND Journal of Economics 29, 324-338.

Christensen, C. M. (1993), „The Rigid Disk Drive Industry: A History of Commercial and Technological Turbulence," Business History Review 67, 531-88. 
Cohen, W. (1995), Empirical Studies of Innovative Activity, in: Stoneman, P., Handbook of the Economics of Innovation and Technological Change, Oxford (UK)/Cambridge (MA), 182-264.

Cohen, W. M. und D. A. Levinthal (1989), „Innovation and Learning: The Two Faces of R\&D,“ Economic Journal 99, 569-96.

Cornelli, F. und M. Schankerman (1999), „Patent Renewals and R\&D Incentives,“ RAND Journal of Economics 30, 197-213.

Czarnitzki, D. und A. Fier (2001), „Do R\&D Subsidies Matter? Empirical Evidence for the German Service Sector,“ Papier präsentiert bei der European Summer School on Industrial Dynamics (ESSID), Cargèse, Korsika.

d'Aspremont,-C. und A. Jacquemin (1988), „Cooperative and Noncooperative R\&D in Duopoly with Spillovers“, American Economic Review 78, 1133-1137.

David, P. (1997), „From market magic to calypso science policy - a review of Terence Kealey's The Economic Laws of Scientific Research,“ Research Policy 26, 229-255.

Davis, S. J., J. Haltiwanger und S. Schuh (1996), „Small Business and Job Creation: Dissecting the Myth and Reassessing the Facts," Small Business Economics 8, 297-315.

Dohse, D. (2000), Regionen als Innovationsmotoren: Zur Neuorientierung in der deutschen Technologiepolitik, Kieler Diskussionsbeiträge 366, 1-30.

Ergas, H. (1987), Does Technology Policy Matter?, in Bruce R. Guile and Harvey Brooks, Technology and Global Industry: Companies and Nations in the World Economy, p. 192.

Gilbert, R. und D. Newbery (1982), „Preemptive Patenting and the Persistence of Monopoly,“ American Economic Review 72, 514-526.

Green J.R. and S. Scotchmer (1995), "On the Division of Profit in Sequential Innovation,“ Rand Journal of Economics 26, 1-17.

Griliches, Z. (1994), „The Search for R\&D Spillovers,“ Scandinavian Journal of Economics 94, Supplement, 29-47.

Griliches, Z. (1998), R\&D and Productivity. The Econometric Evidence, Chicago/London.

Harhoff, D., P. Regibeau und K. Rocket (2001), „Some simple economics of GM food,“ Economic Policy (im Druck).

Harhoff, D. und L. H. Röller (1999), Entwicklung und Perspektiven der bundesrepublikanischen Beihilfepolitik, in: Kaase, M. und G. Schmid (Hrsg.), Eine lernende Demokratie - WZB Jahrbuch 1999. Berlin: edition sigma, 461-498.

Hauff, V. und F. W. Scharpf (1977), Modernisierung der Volkswirtschaft: Technologiepolitik als Strukturpolitik, Köln. 
Hoppe, H. und W. Pfähler (2001), „Ökonomie der Grundlagenforschung und Wissenschaftspolitik," Perspektiven der Wirtschaftspolitik 2, 125-144.

Kealey, T. (1997), „Why science is endogenous: a debate with Paul David,“ Research Policy 26, 897-923.

Klette, T. und D. de Meza (1986), „Is the Market Biased against Risky R\&D?,“ RAND Journal of Economics 17, 133-139.

Klette, T.J., J. Moen und Z. Griliches (2000), „,Do subsidies to commerical R\&D reduce market failures? Microeconomic evaluation studies," Research Policy 29, 471-495.

Klodt, H. (1995), Grundlagen der Forschungs- und Technologiepolitik. Vahlen, München.

Kulicke, M. und U. Wupperfeld (1996), Beteiligungskapital für junge Technologieunternehmen, Heidelberg.

Lach, S. (2000), „Do R\&D subsidies stimulate or displace private R\&D?“, NBER Working Paper, Cambridge, Mass.

Lee, T. und L. Wilde (1980), „Market Structure and Innovation: A Reformulation,“ Quarterly Journal of Economics 194, 429-436.

Lechner, M. und F. Pfeiffer (2001), Econometric Evaluation of Labor Market Policies. Heidelberg.

Levin, R. C. und P. Reiss (1984), Tests of a Schumpeterian model of R\&D and market structure, in: Griliches, Z., R\&D, Patents and Productivity, Chicago, 175-208.

Lessat, V., J. Hemer, T. H. Eckerle, M. Kulicke, G. Licht et al. (1999), Beteiligungskapital und technologieorientierte Unternehmensgründungen: Markt - Finanzierung - Rahmenbedingungen, Wiesbaden.

Leyden, D. P. und A. N. Link (1991), Why are governmental and private R\&D complements?, Applied Economics 23, 1673-1681.

Lichtenberg, F. R. und D. Siegel (1991), The impact of R\&D investment on productivity- new evidence using linked R\&D - LRD Data, Economic Inquiry 29, 203-228.

Loury, G. C. (1979), „Market Structure and Innovation,“ Quarterly Journal of Economics 93, 395410.

Luukonen, T. (1998), The difficulties in assessing the impact of EU framework programmes, Research Policy 27, 599-610.

Mansfield, E. (1996), Contributions of new technology to the economy, in: Smith, B. L. R. und C. E. Barfield, Technology R\&D and the Economy, Washington DC, 114-139.

Mansfield, E., J. Rappoport, A. Romeo, S. Wagner und G. Beardsley (1977), Social and private rates of return from industrial innovations, Quarterly Journal of Economics 
Mayer, M. und R. Müller (1991), Die Deutsche Wagnisfinanzierungs-Gesellschaft mbH (WFG): Erfahrungen und Ergebnisse eines Modellvorhabens, Arbeitspapier, FhG-ISI, Karlsruhe.

Nelson, R. R. (1962, Hrsg.), The Rate and Direction of Inventive Activity, Princeton University Press, Princeton.

Nelson, R. R. und S. G. Winter (1982), An Evolutionary Theory of Economic Change, Cambridge, Mass.

Nordhaus, W. (1969), Invention, Growth and Welfare. Cambridge, Mass.: MIT Press.

Reinganum, J. (1983), „Uncertain Innovation and the Persistence of Monopoly,“ American Economic Review 73, 741-748.

Rüttgers, J. (1996), Innovationsorientierung der Forschungspolitik zur Zukunftssicherung des Standortes Deutschland, Wissenschaftsmanagement 6/1996, 292-296.

Scherer, F. M. (1967), „Research and Development Resource Allocation under Rivalry,“ Quarterly Journal of Economics 131, 359-394.

Schmookler, J. (1966), Invention and economic growth, Cambridge, MA.

Solow, R. (1957), „Technical Change and the Aggregate Production Function,“ Review of Economics and Statistics 39, 312-320.

Spence, M. A. (1984), „Cost Reduction, Competition and Industry Performance,“ Econometrica $52,101-122$.

Stoneman, P. (1995), Handbook of the economics of innovation and technological change, Oxford. 